\title{
Perfil del docente en Bibliotecología en tres universidades de Buenos Aires
}

\section{Adriana Belén Gallardo}

Consejo Nacional de Investigaciones Científicas y Técnicas, Buenos Aires, Argentina

gallardo.a.belen@gmail.com / https://orcid.org/oooo-0oo2-7888-9554

\section{Resumen}

La presente investigación se desarrolló debido a la ausencia de publicaciones sobre el perfil del docente universitario de Bibliotecología y Ciencia de la Información en la Argentina. El objetivo buscado es no solo tener un panorama actual sobre la situación de los profesores en la universidad sino, además, conocer las características de los nuevos horizontes laborales, como así también identificar los problemas y las oportunidades. El análisis se llevó a cabo sobre la población docente de las tres instituciones que otorgan el título de Profesorado en Bibliotecología en Argentina: la Universidad de Buenos Aires, la Universidad Nacional de La Plata y la Universidad Nacional de Mar del Plata. Como resultado se advirtieron un conjunto de características compartidas por los docentes de las tres universidades, lo que evidencia la existencia de un perfil común.

\section{Profile of the professors in Library Science at three universities in Buenos Aires}

Palabras clave

Docente universitario Perfil del docente Bibliotecología Universidades Argentina

\section{Keywords}

\section{Professor} Teacher profile Library Science Universities Argentine 


\section{Introducción}

Si bien existen estudios relacionados con la docencia en Bibliotecología y Ciencia de la Información (ByCI), estos apuntan a los contenidos curriculares y didácticos y no a la determinación de un perfil común para la identificación de problemáticas o posibilidades de capacitación o formación. También se han producido documentos sobre el perfil profesional general del graduado en Bibliotecología pero no sobre la docencia en dicha disciplina.

Del total de 10 universidades que brindan carreras en Bibliotecología solo hay tres instituciones que otorgan el título de Profesorado en Bibliotecología en la Argentina: la Universidad de Buenos Aires, la Universidad Nacional de La Plata y la Universidad Nacional de Mar del Plata.

Teniendo en cuenta lo anteriormente mencionado, una investigación sobre las características de los docentes de Bibliotecología y Ciencia de la Información permite conocer la situación actual de dichos docentes e identificar los problemas y las oportunidades.

\section{Sobre las universidades nacionales que brindan profesorado en Bibliotecología}

La Universidad de Buenos Aires cuenta con la Carrera de Bibliotecología desde el año 1922 pero, a pesar de ello, la formación docente comienza recién en el año 1970. En este primer momento se otorgaba el título de "Profesor en Ciencias de la Información"; desde 1973 a 1993 el mismo recibió la denominación de "Profesor de Bibliotecología y Documentación", para luego pasar a llamarse a partir de 1993 "Profesor de Enseñanza Media y Superior en Bibliotecología y Documentación". Recién desde el último plan de estudios (que data del año 2000) el título otorgado pasa a llamarse "Profesor de Enseñanza Media y Superior en Bibliotecología y Ciencia de la Información".

Se puede acceder al título docente después de graduarse de la Diplomatura en Bibliotecología y haciendo una materia por cada orientación de la licenciatura; dos materias obligatorias de investigación comunes a todas las orientaciones de la licenciatura; las materias del Departamento de Educación "Didáctica General" y "Didáctica Especial" y tres niveles de un idioma latino (portugués, francés o italiano). Se estima que la duración de la carrera docente en Bibliotecología es de 5 años.

A diferencia de otras carreras de la Universidad de Buenos Aires y específicamente de la Facultad de Filosofía y Letras, el Profesorado de Bibliotecología no cuenta con una "Didáctica Especial" sino que debe realizar la de la Carrera de Historia, con contenidos propios de esta disciplina. Además, la didáctica general tiene un marcado enfoque hacia la docencia del nivel secundario, algo que si bien no es errado, no se ajusta específicamente a la docencia de nivel superior que es donde principalmente se desempeña el profesor en Bibliotecología. Nótese que de este modo tampoco se cuenta con una práctica o pasantía en docencia universitaria en el área de Bibliotecología.

Respecto del caso de la Universidad Nacional de La Plata, en esta se creó la Carrera de Bibliotecología en el año 1949 aunque recién se integró como carrera de grado a la Facultad de Humanidades y Ciencias de la Educación en el año 1973. Asimismo, el profesorado se incluyó en el plan de estudios del año 1986 con el nombre de "Profesor en Bibliotecología y Documentación". Actualmente el título recibe el nombre de "Profesor en Bibliotecología y Ciencia de la Información".

Para obtener el título es necesario aprobar las 21 materias que corresponden al título intermedio de "Bibliotecólogo", una materia optativa de una grilla de tres, un seminario, la materia Investigación Bibliotecológica y las materias específicas del 
profesorado: Fundamentos de la Educación, Historia y Política del Sistema Educativo Argentino, Psicología y Cultura en el Proceso Educativo, Didáctica especial de la Enseñanza de la Bibliotecología y Práctica de la Enseñanza en Bibliotecología. La duración estimada del profesorado es de 5 años.

Asimismo, en la Universidad Nacional de Mar del Plata la carrera de Bibliotecario Documentalista se creó en el año 1979, aunque recién se aprobó el Profesorado en Bibliotecología y Documentación en 1984. A diferencia de las otras dos universidades, el profesorado se cursa en el ámbito del Departamento de Ciencias de la Educación de la Universidad Nacional de Mar del Plata y no del departamento de Bibliotecología.

Cabe destacar que, en este caso, la creación del profesorado fue previa al origen de la Licenciatura en Bibliotecología y Documentación de la presente casa de estudios. En la misma universidad se puede obtener, además, el título de Bibliotecario Escolar. Tanto este último, como la Licenciatura se pueden cursar en forma presencial o a distancia; no así el título de Bibliotecario Documentalista y el profesorado que solo se brinda en la primera modalidad.

Para obtener el título es necesario aprobar todas las materias que constituyen el ciclo de Bibliotecario documentalista más las materias del Ciclo Pedagógico propuesto por el Departamento de Pedagogía de la Facultad. Se estima que la duración aproximada de la carrera es de 4 años.

\section{El perfil del docente de Bibliotecología y Ciencia de la Información}

El Diccionario de la lengua española presenta una gran variedad de acepciones para el término "perfil". De entre ellas, el presente trabajo entiende como perfil al "Conjunto de rasgos peculiares que caracterizan a alguien o algo" (Real Academia Española, 2016).

Por consiguiente, en esta investigación, cuando se habla de "perfil" se hace alusión al conjunto de características demográficas, laborales, investigativas y de formación de los docentes en Bibliotecología y Ciencia de la Información. Dentro de cada uno de estos conjuntos se analizarán las variables que los componen. Estas son las que permitirán determinar si existe o no un perfil docente.

La gran mayoría de los estudios previos sobre docentes de Bibliotecología se encargan de analizar las competencias profesionales (Barber, 2005) o el perfil por competencias (Pirela Morillo, 2010). Sin embargo, uno de los principales inconvenientes de esta ventaja del perfil por competencias es el tiempo. Las necesidades cambian continuamente pero, por lo menos en la Argentina, los planes de estudio deben pasar por procesos burocráticos extensos que hacen que una vez implementados ya estén desactualizados. Quizás lo relevado en el perfil por competencias se pueda aplicar más fácilmente a los programas de las materias, un cambio mucho más simple. Un segundo inconveniente es que indicar los conocimientos y competencias básicas que los docentes universitarios de Bibliotecología deberían tener no es lo mismo que establecer si estos viven una realidad en la que esto puede ser posible.

Por consiguiente, antes de establecer las competencias deseables de los docentes de educación superior en Bibliotecología y Ciencia de la Información es necesario conocer su realidad. Para ello, un primer paso es determinar cuáles son sus características actuales y si los diferentes docentes comparten rasgos comunes, es decir, si hay una tendencia que se repite en las diferentes universidades y/o las distintas áreas de enseñanza en las que se divide la Bibliotecología. Una vez determinado esto recién se podrá establecer si esa realidad los habilita a poder alcanzar dichas competencias. 


\section{Estudios sobre las características del docente universitario en Bibliotecolo- gía y Ciencias de la Información en Latinoamérica}

Existen pocas publicaciones previas similares a esta investigación y todas ellas relevan la realidad docente en otros países de Latinoamérica y no en la Argentina. Como primer antecedente se puede mencionar una investigación realizada en el contexto geográfico de México y que se limita a una población de mujeres docentes que además realizan investigación en la disciplina (Licea et al., 2005).

Diez años más tarde se publica un artículo que analiza el contexto brasileño, más específicamente el de la Universidade Federal do Cariri (Silva, Silva y Bernardino, 2015). Lo interesante de este artículo es que estudia la realidad docente en un periodo de 8 años. Así se puede ver la evolución y cambio de algunas de las características a lo largo del tiempo. Entre los datos relevados se encuentran: el nivel de formación, disciplina de origen (porque no todos los docentes tenían formación bibliotecológica), formación docente y experiencia en investigación.

Un artículo más reciente refiere a la realidad colombiana. El estudio se hizo en dos universidades de carácter público (Universidad de Antioquia y Universidad del Quindío) y dos de carácter privado (Universidad de La Salle y Pontificia Universidad Javeriana) (Jaramillo, Salazar Álvarez y Mercado C., 2017). Entre los aspectos relevados se encuentran: modalidad de contratación, género, rango etario, experiencia docente, área de desarrollo disciplinar, la coexistencia de la labor docente con otros trabajos, la formación continua, disciplina de origen, nivel de formación, participación en investigación y formación docente.

Por último, el cuarto artículo refiere a la realidad docente de Escuela Nacional de Biblioteconomía y Archivonomía de México (Cabral Vargas, 2018). Releva aspectos como el género de los docentes, tipo de contratación, dedicación, antigüedad, disciplina de origen, formación docente, participación en investigación y realización de publicaciones. Aunque el desarrollo textual puede servir como base para futuras investigaciones la presentación de la información gráfica genera dudas sobre la precisión de los resultados.

\section{Metodología}

El método utilizado en esta investigación es el cuantitativo. Para el relevamiento de datos se utilizó una encuesta virtual no presencial, a la que los encuestados accedieron por medio de un enlace enviado por correo electrónico junto con una breve explicación sobre la investigación. Se realizó una prueba piloto con 4 personas para determinar la precisión y la claridad de dicho instrumento. La encuesta consistía en un total de 37 preguntas agrupadas en cuatro ejes: el demográfico, el de formación, el laboral, y el de investigación.

Se determinó como población de interés a los docentes de Bibliotecología y Ciencia de la Información que desarrollen actividades en alguna de las tres casas de estudio que brindan el título de profesorado en dicha disciplina. Quedaron excluidos de la población los adscriptos ya que los mismos, de acuerdo con las especificaciones del cargo, no están habilitados a desarrollarse de forma autónoma delante de una clase. De este modo, los cargos de interés para la presente investigación fueron los de: Jefe de cátedra, Profesor adjunto, Jefe de trabajos prácticos, Ayudante de primera y Ayudante de segunda. Cabe aclarar que tampoco se tuvo en cuenta a aquellos docentes que realizan sus tareas a distancia porque este tipo de dictado de clase supone características distintas al resto de los docentes que brindan sus clases de forma presencial. 


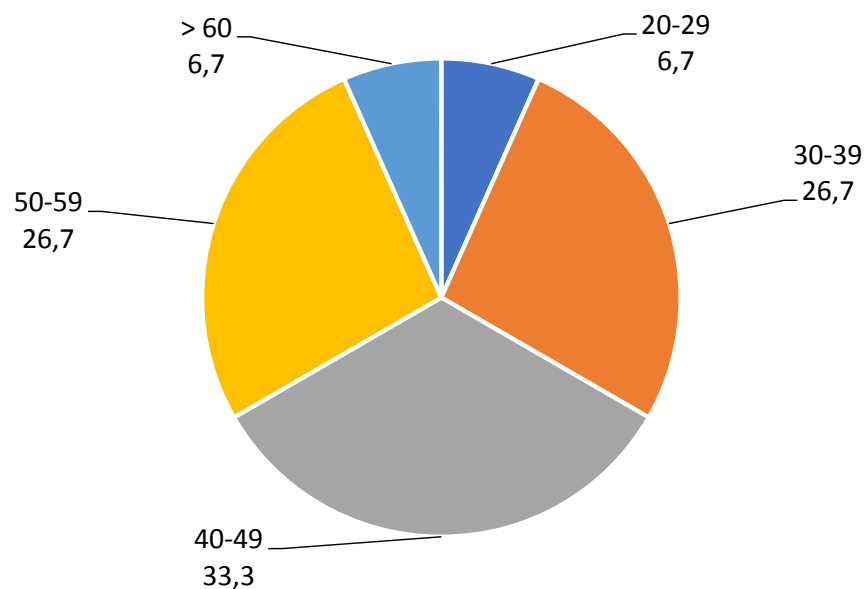

Gráfico 1. Rango etario

De las tres casas de estudio, solo dos, la Universidad de Buenos Aires (UBA) y la Universidad Nacional de La Plata (UNLP), cuentan con información sobre su cuerpo docente en su sitio web. Para obtener la información de la restante, la Universidad Nacional de Mar del Plata (UNMDP), fue necesario contactarse con el Departamento de la carrera. De este modo se pudo determinar que la población total de docentes asciende a 118. Del total 49 de ellos corresponden a la UBA; 28 a la Universidad Nacional de La Plata y 41 a la Universidad Nacional de Mar del Plata.

Como la población resultó ser numerosa se decidió realizar un muestreo aleatorio para asegurar la representatividad de la muestra, el mismo correspondía a un $30 \%$ de la población total. El margen de no respuesta planteado fue de un $5 \%$, es decir, con un $25 \%$ de respuestas se podía dar como validada la encuesta. Debido al carácter estructurado de la investigación, si este número no se alcanzaba, la situación hubiese supuesto la reformulación de la misma.

\section{Resultados}

Fue necesario un recontacto con los docentes de la Universidad de Buenos Aires (diciembre de 2016) y tres con los de la Universidad Nacional de Mar del Plata (diciembre de 2016, marzo y junio de 2017) a través de sus respectivos departamentos. Es así que la primera respuesta fue obtenida el 15/11/2016 mientras que la última fue recibida el 21/06/17. A fines de junio de 2017 se dio por validada la encuesta al sumar un total de 30 respuestas, lo que equivale al $25 \%$ de la población total (mínimo establecido para seguir adelante con la investigación).

\section{Rango etario y género}

En cuanto al rango etario, la mayor población se concentra entre los 40-49 años (Gráfico 1). Sin embargo, si analizamos las distintas universidades por separado, la Universidad Nacional de Mar del Plata es la que tiene docentes mayores: un 60\% se encuentra en el rango etario de 50-59 años, mientras que el $40 \%$ se encuentra en el rango de 40-49. La UNLP y UBA tienen más docentes en el rango 30-39, seguidos por el rango 40-49 (se invierten los dos primeros puestos del promedio). Por último, solo hay dos docentes menores de 30 años.

En cuanto al género (Gráfico 2) coincide la suma del total de resultados con los resultados particulares de cada una de las universidades. 

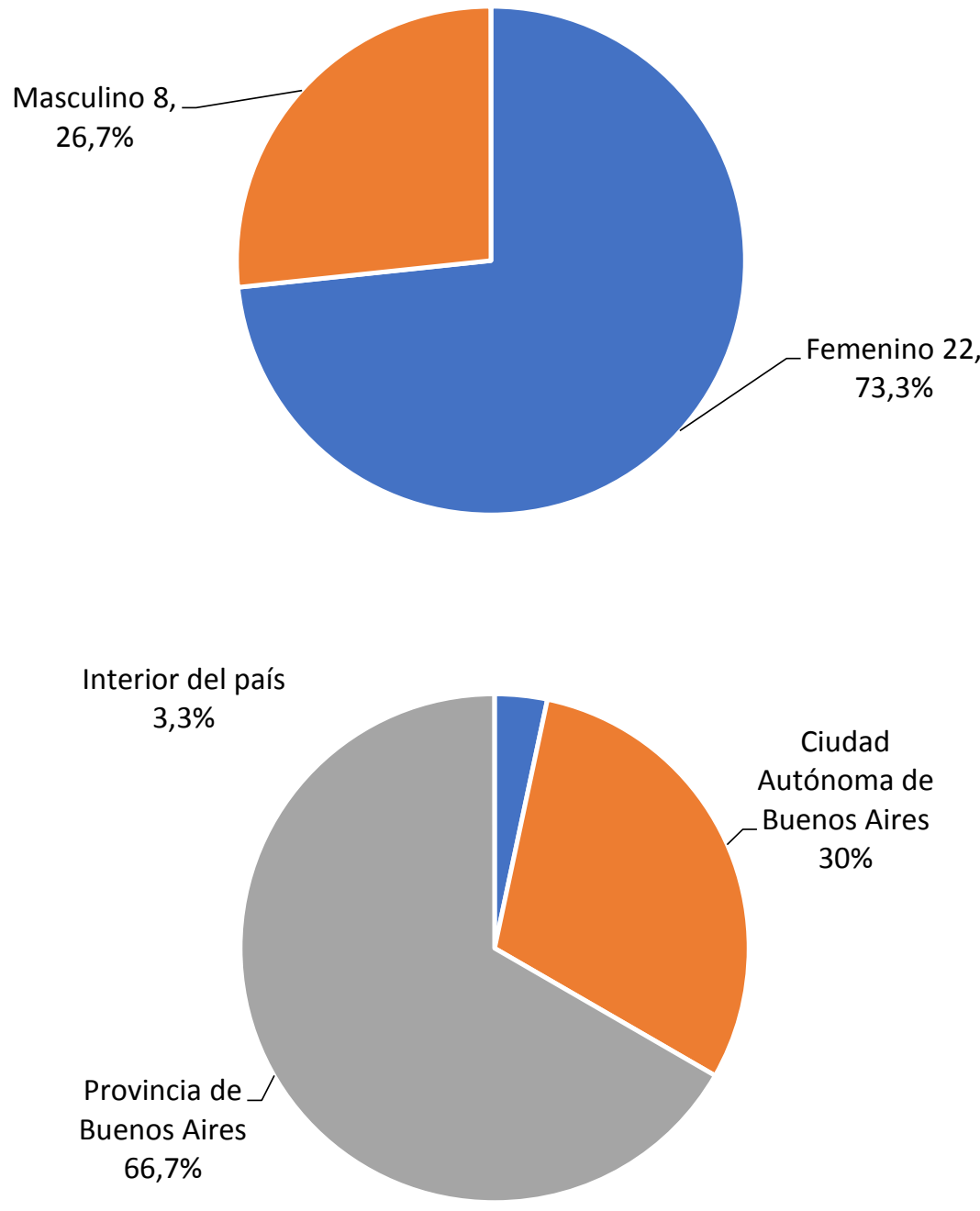

\section{Lugar de residencia, tiempo de viaje y coexistencia con otros empleos}

De los dos tercios de docentes que viven en la provincia de Buenos Aires (Gráfico 3), en su mayoría se trata de docentes que enseñan en la Universidad Nacional de La Plata y la Universidad Nacional de Mar del Plata. Todos aquellos que viven en la Ciudad autónoma de Buenos Aires enseñan en la UBA. Solo hay un docente que vive en Provincia de Buenos Aires y se desplaza hasta CABA para enseñar en la Facultad de Filosofía y Letras.

De los 30 docentes consultados solo 4 viajan hacia su trabajo durante más de una hora (Gráfico 4). Estos datos se oponen a la tendencia en otro tipo de trabajos donde el tiempo de desplazamiento suele ser más largo.

Más del 75\% de los docentes cuentan con más de un empleo (Gráfico 5). Seis de los siete docentes que no cuentan con un trabajo adicional, dan clases en la Universidad Nacional de Mar del Plata. Los seis son docentes concursados y tienen dedicación exclusiva. Además, solo uno de esos seis cuenta con más de un cargo docente.

De ese porcentaje de docentes con más de un empleo, el 91\% trabaja en otras bibliotecas. Solo hay tres que realizan otro tipo de trabajos: investigación en CONICET, 

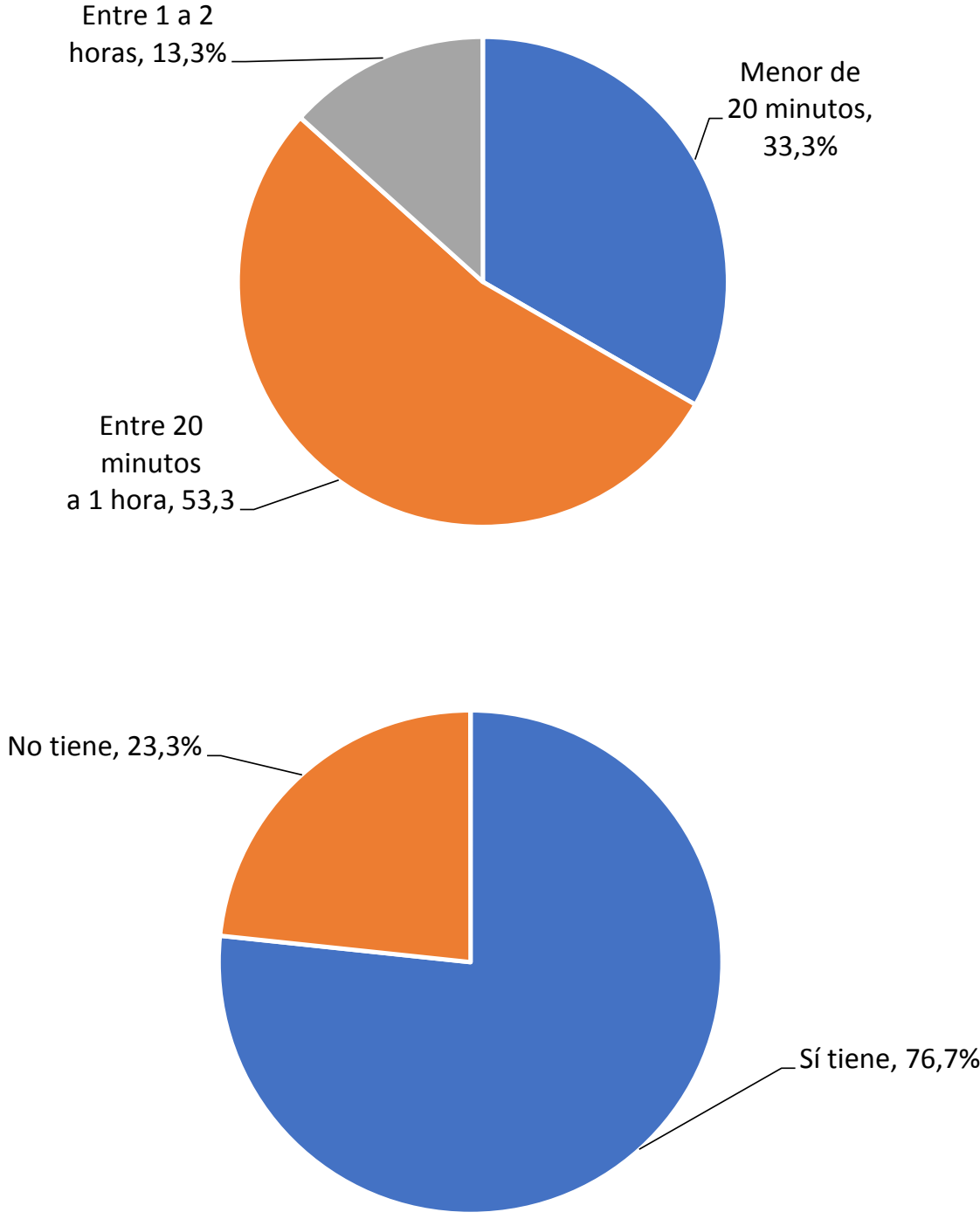

gestión editorial y desarrollos informáticos. En la misma línea, el 83\% de los encuestados manifiesta que sus diferentes trabajos están muy relacionados entre ellos. Solo un $13 \%$ piensa que esa relación es un poco menor.

\section{Formación}

Es una tendencia que se repite en las diferentes universidades el hecho de que la formación mínima alcanzada sea la Universitaria de grado (Gráfico 6). Solo dos docentes tienen formación universitaria de pregrado. Se podría pensar que estos dos docentes son jóvenes y por lo tanto continúan con su formación universitaria pero se encuentran dentro del rango etario de 40-49 años. Un total de 13 docentes tienen formación de posgrado: 9 de maestría y 4 de doctorado. Solamente 3 de los 13 docentes con nivel de posgrado son de la Universidad de Buenos Aires y el máximo nivel alcanzado por ellos es el de Maestría.

Igualmente es importante resaltar que la situación del profesional en Bibliotecología es complicada en cuanto a posgrados, porque en el país la oferta de posgrados especializados en la disciplina es reducida ${ }^{1}$. Por lo tanto, la formación de dicho nivel debe
Gráfico 4. Tiempo de viaje hacia el trabajo

Gráfico 5. ¿Cuenta con otro empleo además de la docencia?

1. UBA otorga el título de Doctor de la Universidad de Buenos Aires (Área: Bibliotecología y Documentación) y durante los años 2016 y 2017 se dictó la Maestría en Bibliotecología y Ciencia de la Información en colaboración con la Biblioteca Nacional Mariano Moreno. 
Gráfico 6. Último nivel educativo alcanzado

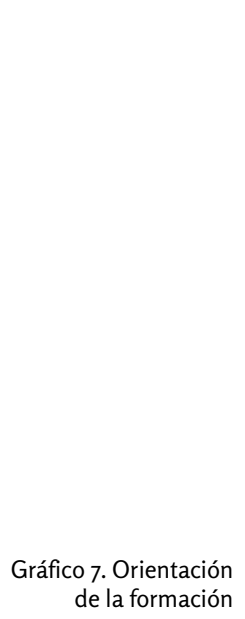

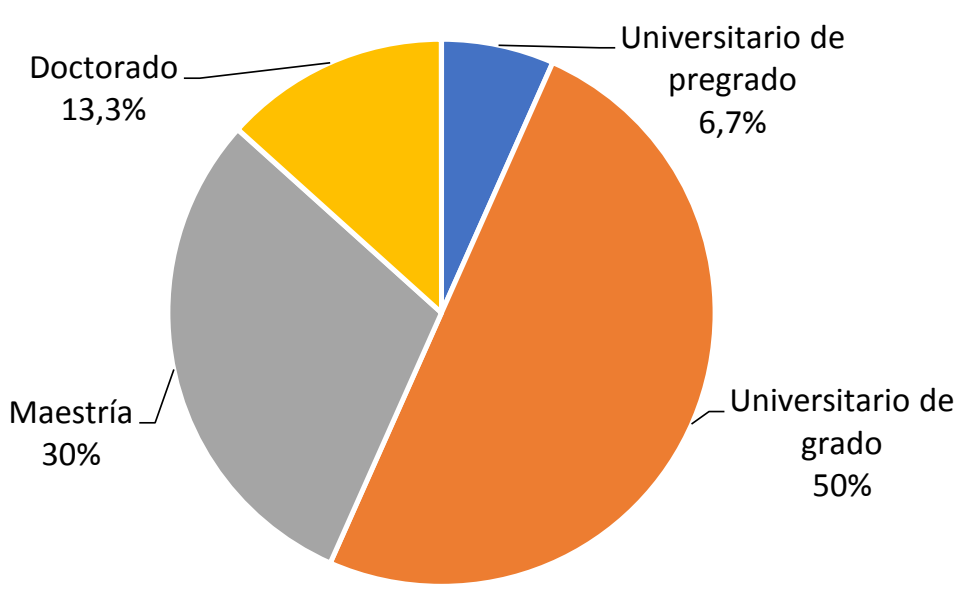

Procesamiento de la información

Recursos y servicios

Formación general/sin división por orientaciones

Gestión de Unidades de Información

Metodología

Archivología

Preservación y Conservación

Tecnología de la Información

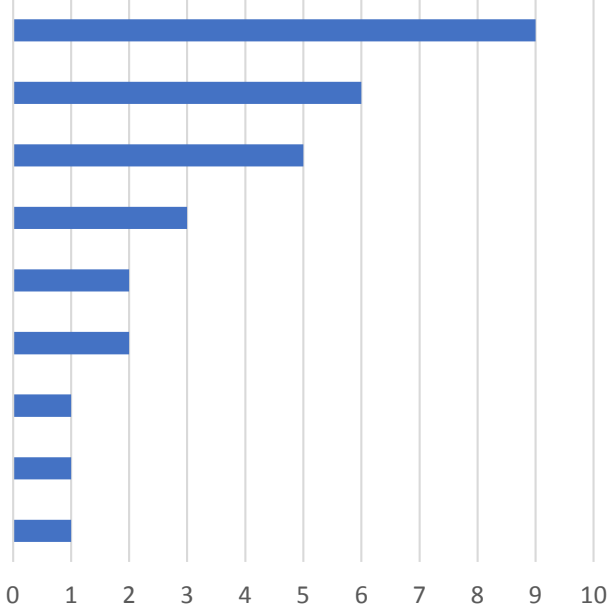

realizarse a distancia o viajando a otros países y además se encuentran valuadas en moneda extranjera, lo que hace la situación aún más difícil El único docente con una formación distinta es Ingeniero en Informática. Se podría pensar que con dicho título se encuentra a cargo de una materia de la orientación "Tecnología" pero es docente de una materia de "Gestión". Uno de ellos además tiene formación en Archivología. Como dato adicional, todos los docentes que respondieron se formaron en la misma institución en la que hoy dan clases.

Con relación a la distribución de docentes formados en las distintas orientaciones dentro de la carrera, predomina el área de "Procesamiento de la información" (Gráfico 7), tendencia que se repite en las tres universidades. El 20\% declara haberse formado en carreras que cuentan con distintas orientaciones pero cuya especialización elegida no aparece listada entre las opciones brindadas en la encuesta. Las opciones que le siguen son las formaciones generales (sin división de orientaciones) y "Recursos y servicios". 


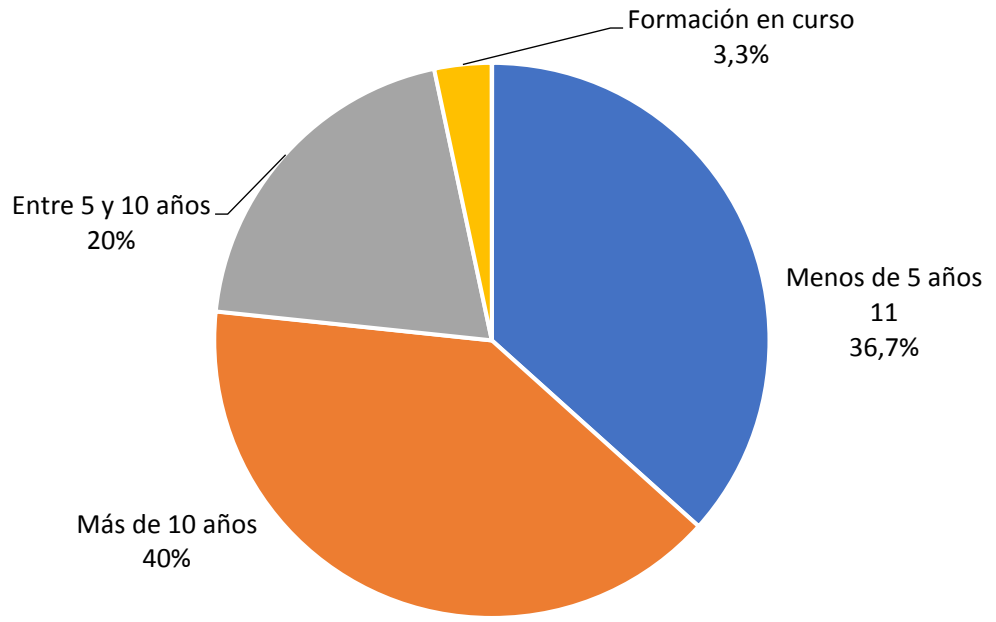

Gráfico 8. Tiempo transcurrido desde el fin de la formación de grado o pregrado

Respecto al tiempo que transcurrió desde la finalización de sus estudios de grado (Gráfico 8) se puede observar que conviven principalmente dos generaciones de graduados: la graduada más recientemente hace menos de 5 años (compuesta principalmente por un rango etario de 30-39 años) y la graduada hace más de diez años (compuesta principalmente por un rango etario de 40-49 años). En la Universidad de Buenos Aires predomina la más "joven"; en la Universidad Nacional de Mar del Plata la más longeva; mientras que en la Universidad Nacional de La Plata ambas están prácticamente distribuidas por igual.

Todos los docentes manifestaron que siguen formándose. El método de capacitación más elegido es la asistencia a Seminarios y Congresos. El 70\% de los encuestados realizó o está realizando posgrados. Le siguen en popularidad los cursos de actualización profesional y la formación autodidacta. A los docentes se les dio las cuatro opciones de formación mencionadas, todas recibieron entre un $60 \%$ y un $80 \%$ de selecciones. Esto quiere decir que la mayoría de los docentes combinan más de un método de formación profesional adicional a su formación de grado o pregrado.

\section{Formación docente}

Solo 12 docentes no tienen ningún tipo de formación docente o didáctica. Del resto de los encuestados, la mayoría ya concluyó sus estudios (Gráfico 9). La distribución de docentes con formación en docencia es la siguiente: de la Universidad de Buenos Aires son dos, en la Universidad Nacional de La Plata son nueve y en la Universidad Nacional de Mar del Plata, siete.

En cuanto a los tipos de formación docente (Gráfico 10), el Profesorado de la Universidad de Buenos Aires solo ha sido realizado por un único docente (graduado de la misma casa de estudios). Quizás esto se deba a la inadecuación de la carrera que se ha indicado al principio del presente trabajo.

Si en cambio se analiza por separado cada Universidad, predomina la selección del Profesorado de la propia casa de estudio y esta es una tendencia que se repite a lo largo de las tres. 


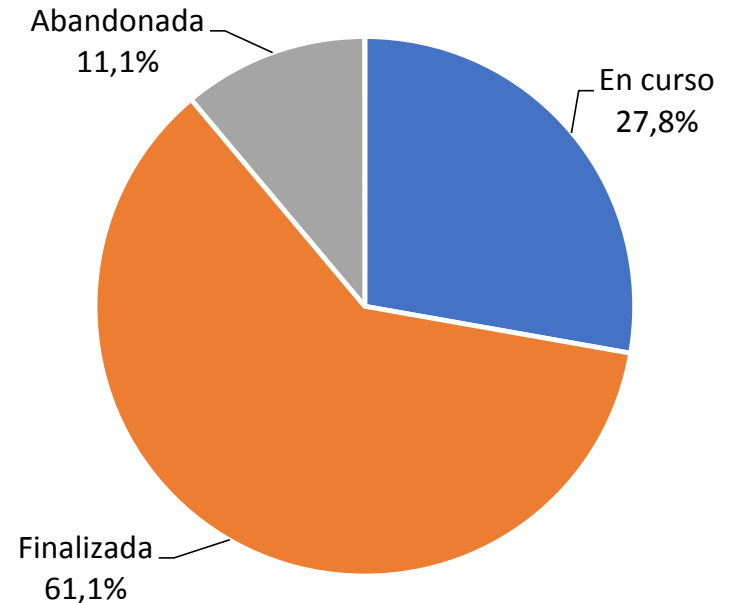

Profesorado en Bibliotecología y Ciencia de la Información de la UNLP

Otra

Cursos de formación docente

Profesorado en Bibliotecología y Documentación de la UNMDP

Profesorado en otra disciplina

Profesorado de Enseñanza Media y Superior en Bibliotecología y Ciencia de la Información de la UBA

\section{Labor docente}

La mayoría de los encuestados son Profesores Adjuntos y solo hay un Ayudante de $2^{a}$ (Gráfico 11).

Se entiende por docentes concursados o regulares aquellos que han sido seleccionados por un jurado, mientras que los interinos son aquellos que han sido propuestos por el director y aceptado por el consejo directivo. Más de la mitad de los encuestados son docentes concursados o regulares (Gráfico 12). Sin embargo, si se analizan las universidades por separado se notan ciertas diferencias: en la UNLP todos -salvo un docente- son concursados, en la Universidad de Buenos Aires la mayoría son interinos, mientras que todos los de la UNMDP son concursados.

En cuanto a la dedicación el 56\% (17 docentes) cuenta con una dedicación simple (10 horas semanales), 7 cuentan con dedicación exclusiva (40 horas de docencia e investigación) y 6 con semiexclusiva (2o horas semanales). Al analizar las universidades de forma aislada se puede ver que la tendencia general no se observa en la Universidad Nacional de Mar del Plata (Gráfico 13). Por otro lado, a diferencia de otras carreras de estas y otras universidades argentinas, todos los docentes encuestados son rentados, es decir no hay ninguno que desarrolle sus tareas ad honorem. En cuanto al área de desempeño si bien se puede observar una predominancia de las áreas de 

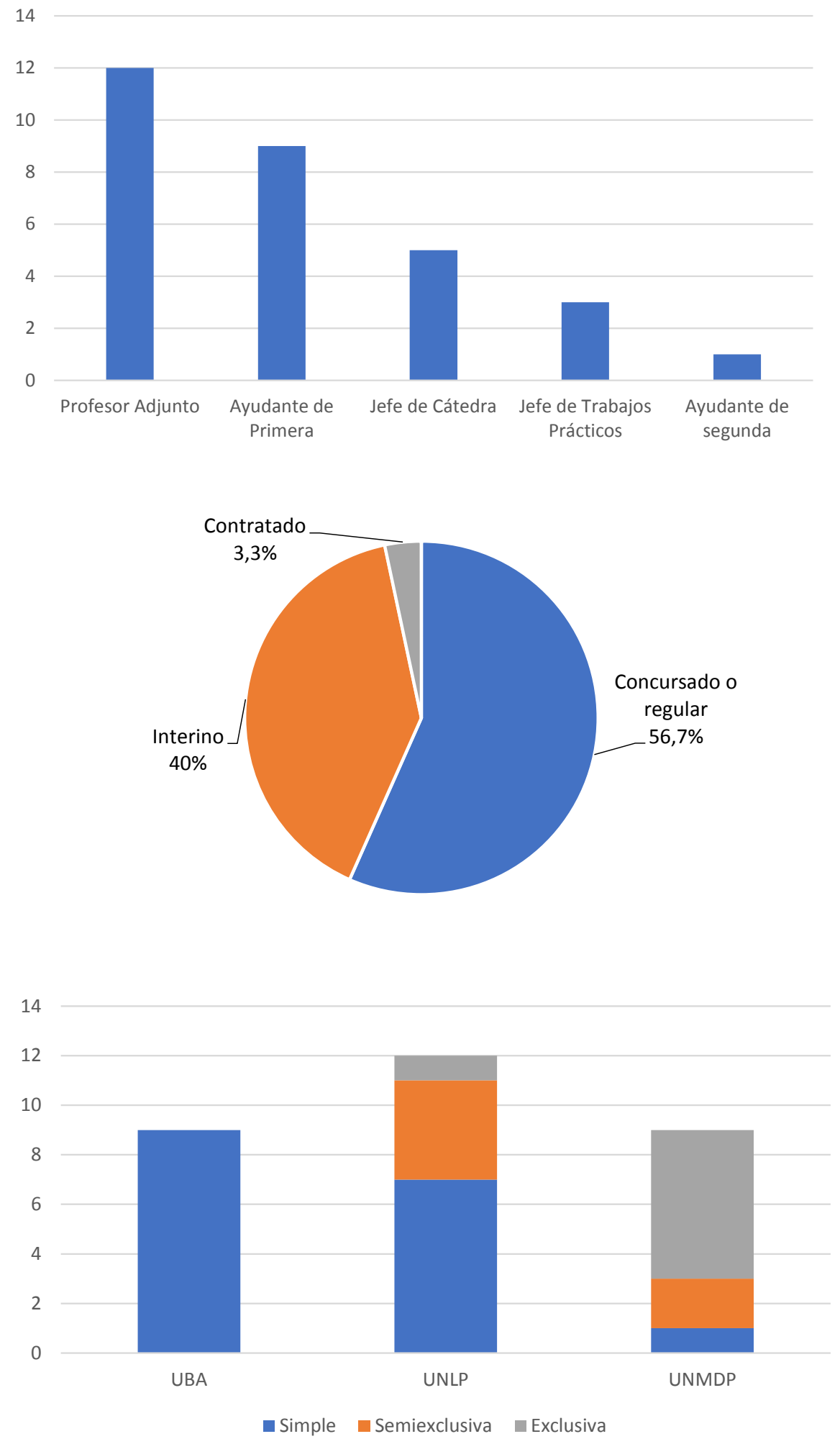

"Procesamiento de la información" y "Gestión de unidades de información" (Gráfico 14), el análisis por universidad refleja que en la Universidad de Buenos Aires hay más docentes en el área de Procesamiento; en la Universidad Nacional de La Plata predomina Gestión, mientras que en la Universidad Nacional de Mar del Plata hay un foco en las materias de Metodología.
Gráfico 12. Tipo de categoría docente

Gráfico 13. Dedicación docente en cada universidad 

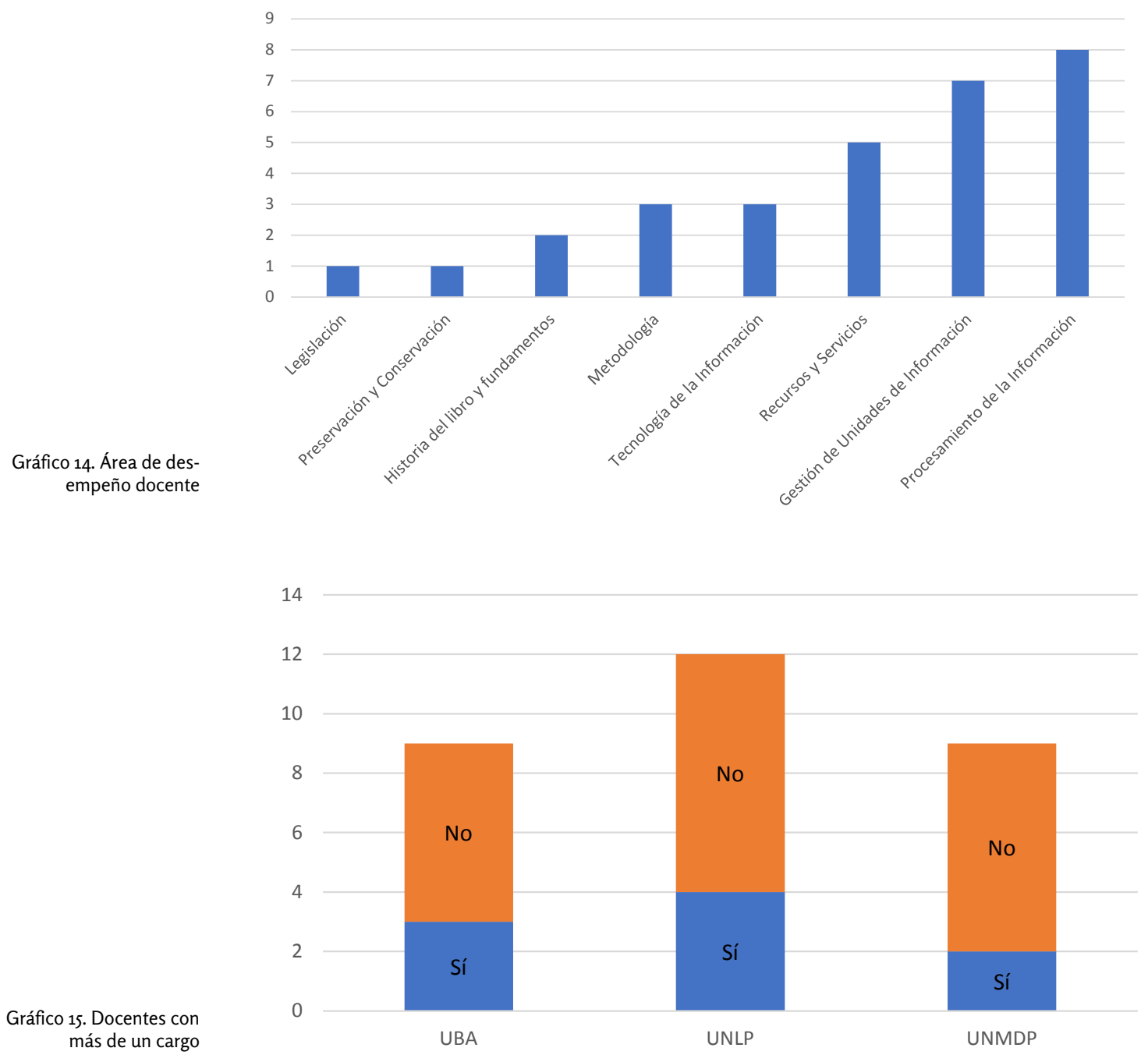

Más del $75 \%$ de los docentes solo cuenta con un cargo docente. Esta tendencia se repite en las tres universidades (Gráfico 15).

Cabe aclarar que en la Universidad Nacional de Mar del Plata, en cuyos docentes predomina la dedicación exclusiva, al momento de realizar el relevamiento existía una ordenanza del Consejo Superior que le impedía a estos contar, además, con una dedicación simple.

Se podría pensar que no se suele tener más cargos debido a que se dedica el tiempo a otros trabajos externos a la facultad. Sin embargo, en base al análisis de los datos recabados se pudo observar que, en general, tanto aquellos docentes que tienen un solo cargo como aquellos que tienen más de uno, ambos suelen tener otros trabajos por fuera de la docencia (Gráfico 16).

Más de la mitad de los docentes con más de un cargo docente cuenta con dos de ellos (Gráfico 17). Tres de las cuatro personas que cuentan con más de dos cargos también tienen un trabajo por fuera de la docencia. 


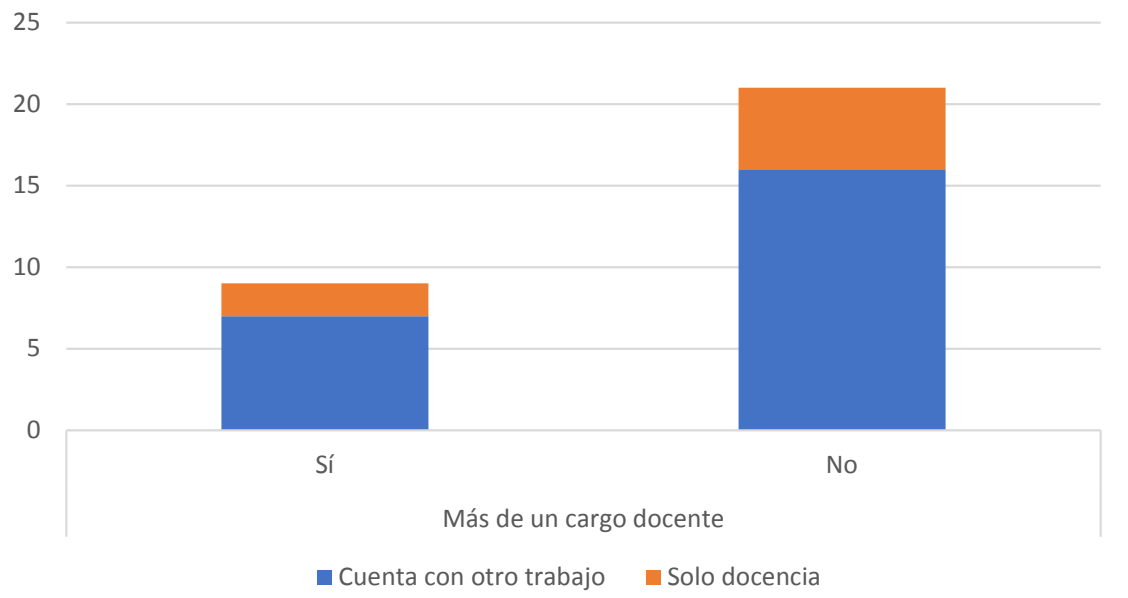

- Cuenta con otro trabajo Solo docencia

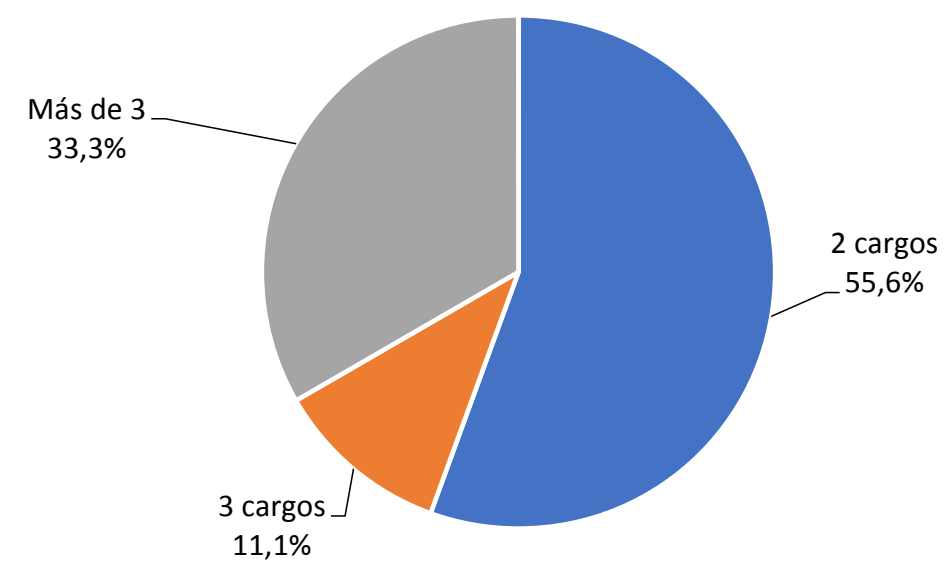

En cuanto a la antigüedad en el cargo se puede observar que la tendencia en las tres universidades es la existencia de cargos con más de 10 años de antigüedad (Gráfico 18). Los cargos de menos de 5 años son pocos, lo que permite advertir que no está habiendo un recambio generacional de los docentes. No se puede saber a ciencia cierta si esto se debe a una falta de interés de las nuevas generaciones para el ingreso a la docencia o los mecanismos de ingreso no brindan las facilidades para que ingresen nuevos profesionales.

\section{Asociaciones profesionales, investigación y participación en eventos}

Más de tres cuartos de los encuestados no forma parte de asociaciones profesionales. De los que respondieron afirmativamente dos pertenecen a la Asociación de Bibliotecarios Graduados de la República Argentina, otro al Colegio de Bibliotecarios Graduados de la Provincia de Buenos Aires y un tercero a la Asociación de Bibliotecarios Jurídicos. Por último, otros dos pertenecen a asociaciones Internacionales o de otros países.

\section{Gráfico 16. Relación entre cantidad} de cargos y otros trabajos
Gráfico 17. Cantidad de cargos docentes 
Gráfico 18. Antigüedad en la docencia

Gráfico 19. ¿Cuándo ha realizado investigaciones?
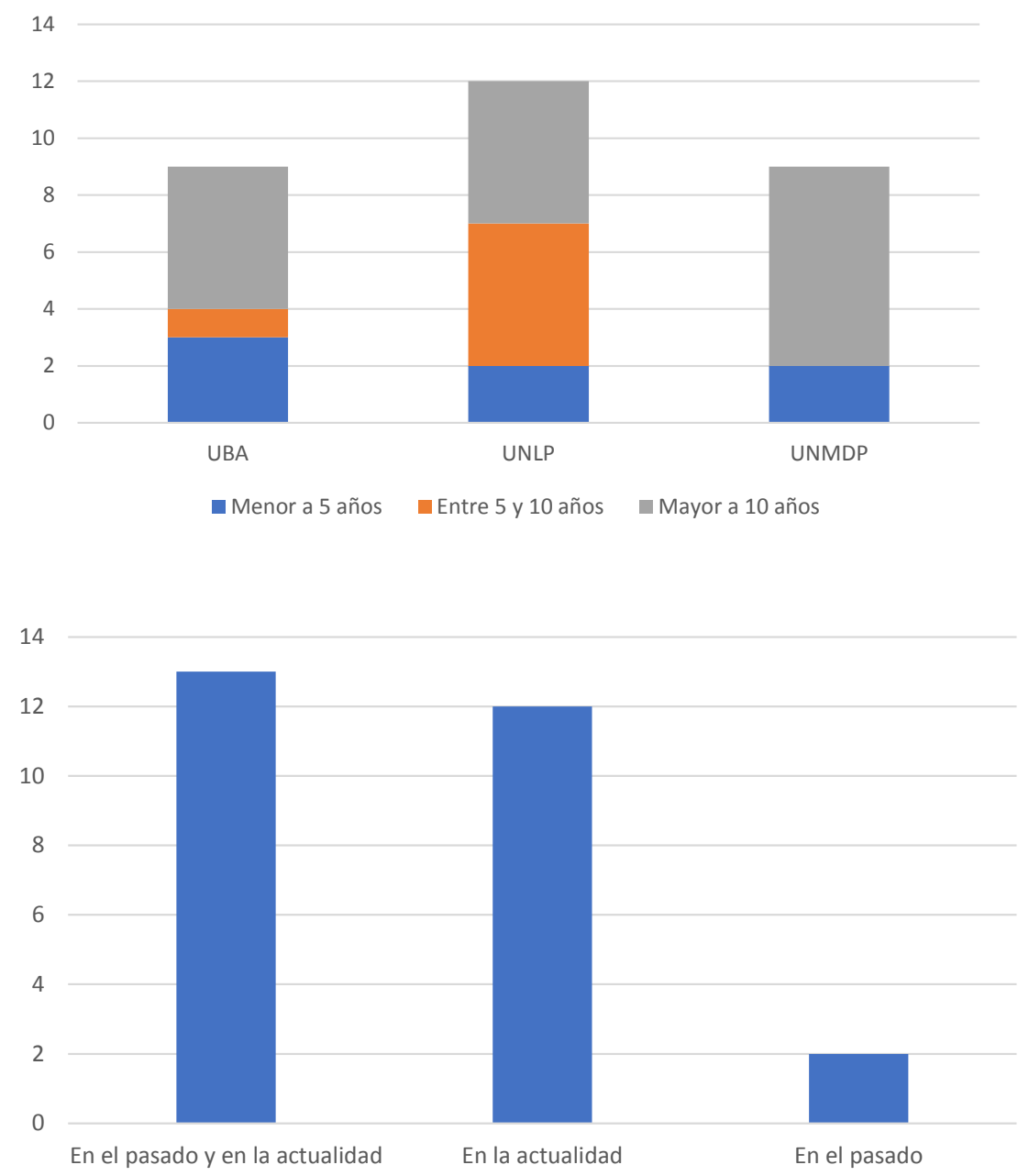

En cuanto a la investigación solo tres docentes no han realizado ni están realizando algún tipo de investigación. Los tres tienen una formación reciente, dos de ellos terminaron su formación universitaria hace menos de cinco años y el tercero todavía la está cursando. Sin embargo, dos de ellos han manifestado su interés por la investigación en un futuro cercano.

De aquellos que sí han realizado o están realizando investigaciones, todos menos uno quieren continuar haciéndolo. La gran mayoría de los docentes, más precisamente 25 de un total de 27, están investigando en la actualidad (Gráfico 19).

Nótese que de estos 25 docentes que están investigando actualmente, 7 de ellos tienen dedicación Exclusiva, que es aquella que por definición tiene como tarea inherente la investigación y 2 Semiexclusiva en la UNMDP donde también se les exige a los docentes con este tipo de dedicación la dirección de grupos de investigación (Gráfico 20). Es así que la mayoría de los docentes encuestados que investigan en la actualidad tienen dedicación simple ( 6 tanto en la UNLP como en la UBA), la cual no les exige el desarrollo de investigaciones. En la misma situación están los docentes que cuentan con dedicación semiexclusiva por fuera de la UNMDP, en este caso, cuatro docentes de la UNLP. 


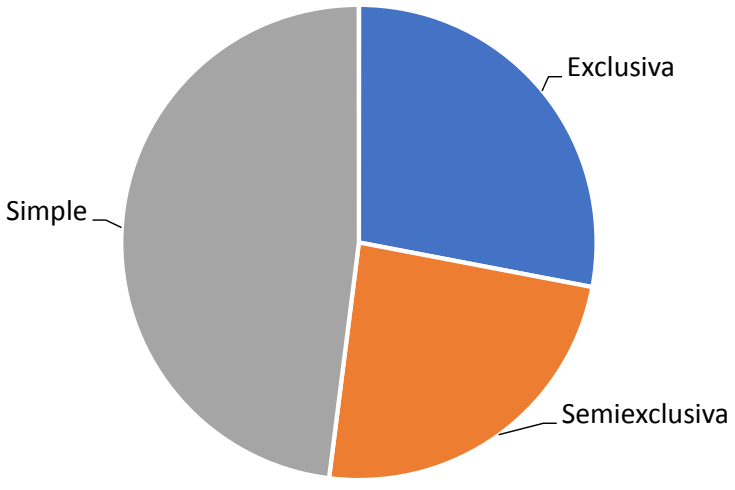

En relación a las publicaciones, solo cuatro docentes no han realizado ningún tipo de publicación. El tipo de publicación más realizado, alcanzando un total de $89 \%$, son artículos en revistas académicas. Le siguen los capítulos de libros y artículos en revista de difusión. Solo un $30 \%$ ha escrito libros. A pesar de ser un tipo de documento poco común, 5 docentes han escrito en diarios. Por otro lado, solo un docente no ha presentado ponencias en ningún tipo de evento.

\section{Opiniones sobre la situación actual de la docencia en Bibliotecología y temas relevantes}

Al final de la encuesta se incluyó una pregunta abierta para relevar la percepción de la situación docente en Bibliotecología. En las respuestas se pueden observar varios temas recurrentes:

Esta pregunta tenía un doble objetivo, primero identificar si cada profesor conocía la situación general de la realidad docente o solo la que se vive en su institución y, por otro lado, qué percepción tenía de la misma (si es que contaba con información suficiente). Si bien la mayoría de las respuestas permiten entender que el docente en $\mathrm{ByCI}$ conoce la realidad de la docencia en la disciplina, en los testimonios se puede observar que algunos docentes no están al tanto de la misma. Sin embargo, no es claro si su desconocimiento se debe a que no hay información disponible o a que no hay interés en los profesores en acceder a ella. Dos ejemplos de ello son los siguientes:

Resulta complejo pensar en un panorama general sin contar con información relativa a otros contextos (otras universidades, institutos terciarios, etc.).

Considero que no cuento con un conocimiento general sobre la situación docente en Bibliotecología. Me gustaría contar con algo más de información para responder.

Otros profesores, en cambio, sostienen que las diferencias son visibles y, en palabras de unos de ellos, piensan que la formación:

Es muy dispar dependiendo del tipo de institución en la que se han formado los docentes, de sus inquietudes personales por mejorar su labor frente a los alumnos y de las políticas que al respecto asumen las instituciones educativas para las cuales trabajan.
Gráfico 20. Dedicación de los docentes que realizan investigaciones 
Se reconoce que la enseñanza general y, específicamente, la formación docente no es la correcta tanto porque no incluye contenidos adecuados para nuestra disciplina como así también porque aquellos que sí los tienen están obsoletos. Son numerosos los comentarios que sostienen esto y que, además, la situación permanece así debido al desinterés no solo de las autoridades sino también de los propios docentes:

Falta de incentivos para el desarrollo de la investigación, y falta de la actualización de los planes de estudio y mayor compromiso político con la disciplina.

Hay poca formación continua, poco interés por capacitarse y mantenerse actualizado. No se hace énfasis en esto en la universidad.

Es una carrera muy prometedora pero, al menos en la Argentina, la formación resulta muy deficitaria. Los contenidos escasamente ofrecen la formación que los bibliotecarios requieren para su ejercicio profesional. Por otro lado, el nivel docente es deficitario; entiendo que en un alto porcentaje no son precisamente aquellos mejor formados y especialistas en las diferentes temáticas.

[...] los contenidos no son acordes a lo que requiere la práctica profesional, en relación al rol que el profesional de la información tiene en la actual sociedad del conocimiento. Las clases magistrales y las evaluaciones no conducen a la reflexión y el pensamiento crítico que se pretende que el estudiante logre.

Sin embargo, algunos docentes están realizando acciones para producir cambios:

En la UNLP estamos trabajando fuertemente para repuntar la carrera del profesorado de Bibliotecología y Ciencia de la Información desde las cátedras Didáctica especial y Prácticas de la enseñanza en Bibliotecología. Este año, por ejemplo, hemos puesto en marcha un Laboratorio docente. El 3 de diciembre tendremos el 6to. encuentro del año. Está pensado para docentes y adscriptos de las carreras de la Lic. y Prof. de Bibliotecología de la UNLP, para aquellos que ejercen la tarea docente pero que no son profesores recibidos, sino licenciados o adscriptos alumnos. Trabajamos sobre temas de interés pedagógico y didáctico, sobre las planificaciones de nuestras clases.

Tengo una visión optimista en esta Universidad, luego de varios años de militancia desde la comisión de estudiantes y en junta asesora hemos logrado reivindicar el profesorado de Bibliotecología y contamos hoy con profesores egresados. Asimismo en el último año se canalizaron las preocupaciones por parte de los ayudantes en ejercicio por mejorar métodos y estrategias de enseñanza.

También se manifiesta en muchos de los comentarios la necesidad de constante formación complementaria debido a que nuestra disciplina está en constante mutación:

Estamos en un proceso de reformulación de la profesión que debe trasladarse a la formación de los futuros profesionales.

Desde mi punto de vista, el docente debe capacitarse constantemente y mantenerse en la línea de lo nuevo.

Para tener en cuenta la visión docente en Bibliotecología, hay que (re)considerar tres características: la docente, que se debe adaptar al nuevo entorno mutable; a la investigación, que debe ser más tangible a la situación social de cada comunidad y la de extensión, que es la labor con la comunidad y que no debemos olvidar de considerarla dentro de los procesos educativos. 
Considero que estamos ante un momento de muchos cambios, especialmente en el sector de procesos técnicos, en el que desempeño mi actividad docente. Se hace patente la necesidad de tener una actualización constante de los conocimientos y hacer un seguimiento de los nuevos temas que surgen en el área, para poder mantener los contenidos de las materias actualizados y ser solvente como docente durante las clases.

Otra cuestión recurrente en los comentarios es que el ingreso a la docencia es muy difícil. Faltan vacantes, los requisitos de ingreso son muy exigentes y los métodos no son los adecuados. Hay muchos docentes interinos debido a que no se abren concursos. Se observa la necesidad de recambio para traer nuevos puntos de vista y contenidos más actualizados. Los pocos que pueden ingresar, en general tienen cargos inadecuados: realizan tareas que no se condicen con las incumbencias del cargo, es decir, están realizando tareas de un puesto superior al que tienen:

Es una carrera de largo aliento, el ingreso en el nivel universitario se ve retrasado por falta de vacantes, requisitos exigentes, salarios bajos, alta exigencia bajo la figura de docente-investigador/a, etc. La concepción actual de carrera profesional parece venir a resolver el riesgo de perder la fuente laboral por el llamado a concurso cada 7 años, periodicidad que, no obstante cabe aclarar, no siempre se cumplía. Supone un sistema de evaluación cada 4 años que puede resultar productivo siempre y cuando se implemente con criterio y en aras de favorecer la performance docente, solapada muchas veces por la investigación.

Los cargos suelen ser interinos varios años, y recién ahora los docentes tenemos estabilidad laboral. En la UNLP, los ayudantes de primera están a cargo de una comisión (sin el acompañamiento presencial del titular), preparan y corrigen trabajos y exámenes, es decir, cumplen algunas funciones de JTP (cargos que no existen)

Sería deseable que los docentes accedieran a los concursos no más allá de los 4 años de ejercicio de la docencia.

También provoca problemas la poca oportunidad para iniciarse en la docencia.

[...] no se logra completar los equipos docentes en las distintas asignaturas.

Otro de los aspectos que es mencionado por más de un docente es la falta de reconocimiento social y la necesidad de consolidar la profesión.

Falta mucho reconocimiento social, valoración de sus profesionales y definición del rol de la biblioteca en la sociedad actual.

[Se] necesita consolidar su profesionalización en el campo laboral.

Este desconocimiento del rol del bibliotecario por parte de la sociedad y las autoridades lleva a que haya pocos ingresantes, los cargos disponibles sean escasos y mal pagos y que tareas que pueden ser realizadas por bibliotecarios sean efectuadas por profesionales de otras disciplinas, tal como lo indica uno de los docentes encuestados:

Hay muchas materias vinculadas al uso criterioso de la información y de las tecnologías que están dictando informáticos y que los profesionales de la información estamos capacitados plenamente para dictar. 
Por último, muchos de los comentarios refieren a la importancia de la participación en la investigación del docente en Bibliotecología. Uno de los reclamos principales es que:

El docente pueda tener un mejor posicionamiento salarial de forma tal que pueda invertir más horas en investigación y formación en posgrados.

Otra crítica refiere a la necesidad de conocer a cada comunidad atendida por medio de la investigación. Por último, se evidencia que hay una "falta de incentivos para el desarrollo de la investigación".

\section{Conclusiones}

\section{Rasgos compartidos que determinan la existencia de un perfil común}

En cuanto a las características sociodemográficas se puede observar que en las tres universidades hay predominancia de docentes del género femenino, tal como se indicó más arriba, repitiendo la tendencia de género de los estudiantes de la carrera de Bibliotecología y Ciencia de la Información. Otro aspecto compartido es que la gran mayoría de docentes viven en zonas geográficas cercanas a la universidad donde brindan clases, lo que se relaciona con que también se comparta entre los diferentes encuestados un tiempo medio de viaje hacia el trabajo de entre 20 minutos y una hora. La cercanía de la vivienda con el lugar de trabajo puede vincularse con el hecho de que muchos de los docentes relevados cuentan con otro trabajo por fuera de la docencia y por lo tanto necesitan ganar el mayor tiempo posible para poder desempeñarse en ambos trabajos. Además, es importante resaltar que incluso aquellos que poseen más de un cargo docente cuentan con este trabajo adicional por fuera de la enseñanza. Cabe agregar que este segundo trabajo se desarrolla en casi todos los casos en bibliotecas.

En relación con la educación, en general, el título mínimo alcanzado es el Universitario de Grado. Si se tiene en cuenta el título más reciente, los rangos etarios más avanzados son aquellos que han realizado o están realizando formación de posgrado. Los docentes se gradúan en las mismas casas de estudio en las que más adelante dan clases. Muchos docentes tienen una formación general (no dividida en orientaciones) pero, si se tiene en cuenta al resto, predomina la elección de la orientación en "Procesamiento de la Información". Se observó que existe una formación constante. El método más elegido es la asistencia a eventos científicos. Sin embargo suelen combinarse más de un método de formación complementaria.

Si se tiene en cuenta la actividad docente, salvo raras excepciones, los docentes siempre están formados en Bibliotecología y Ciencia de la Información, es decir, no suelen encontrarse docentes que brinden clases en la carrera y provengan de otras disciplinas. La mayor cantidad de docentes brindan clases en las áreas de "Procesamiento de la Información" (lo que no es raro teniendo en cuenta que es la orientación de formación más elegida entre los profesores de la encuesta), "Recursos y servicios" y "Gestión". Sí se analiza el tiempo que ha pasado desde que han terminado su carrera de grado se observa que conviven dos generaciones de docentes: los graduados hace menos de cinco años y aquellos que han finalizado su formación básica hace más de 10 años. Si se tiene en cuenta el aspecto laboral de la docencia, lo que predomina es la dedicación simple.

No es una tendencia común la participación en asociaciones profesionales. Son muy pocos los docentes que lo hacen. En cambio, la participación en investigaciones (en el pasado o en la actualidad) se observa en una amplia mayoría. Hay, además, un 
marcado interés en seguir haciéndolo. Por último, se observó incluso aquellos que no están obligados en base a su dedicación docente y los estatutos de sus respectivas universidades están investigando o lo han hecho en el pasado.

\section{Rasgos en que se distinguen entre sí los docentes de las distintas universidades}

El primer rasgo distintivo es la edad: mientras que los docentes de la Universidad Nacional de Mar del Plata tienen en su mayoría entre 50-59 años; los de las universidades restantes tienen en promedio entre 30-39 años. En esa misma línea, los docentes de la primera se han graduado hace más tiempo (más de 10 años) mientras que en la Universidad de Buenos Aires, el promedio es de graduados más recientes (menos de 5 años). Por otro lado, en la Universidad Nacional de La Plata, conviven ambas generaciones de graduados. La edad de ingreso y permanencia en la docencia depende estrictamente de las políticas de los respectivos departamentos: dependen de los requisitos que se exigen para ingresar, de los lapsos de tiempo máximos de permanencia en un cargo antes de un nuevo llamado a concurso, de las facilidades de los diferentes mecanismos de ingreso, etc. Las diferencias encontradas, se relacionan con cómo estos aspectos varían en cada una de las universidades.

Otro factor que obtuvo resultados distintos en las diferentes casas de estudio es la capacitación docente. Esta es casi nula en la Universidad de Buenos Aires, mientras que es alta en la Universidad Nacional de La Plata y la Universidad Nacional de Mar del Plata. Quizás esto tenga que ver con que en estas dos últimas universidades, el profesorado en Bibliotecología y Ciencia de la Información está más consolidado y que es específico de ByCI.

El último aspecto en que difieren los resultados tiene que ver con el modo de contratación y los cargos. Mientras que en la Universidad de Buenos Aires la mayoría de los docentes son interinos, en la Universidad Nacional de Mar del Plata y la Universidad Nacional de La Plata los docentes han ingresado mediante concursos. La apertura de concursos en la Universidad de Buenos Aires es muy difícil de alcanzar, lo que explica que la mayoría de sus docentes sean interinos. Además, si se tiene en cuenta que la población de las otras dos universidades lleva muchos más años en la docencia, se desprende que los concursos se han realizado hace mucho más tiempo, donde la realidad universitaria era diferente.

Por otro lado, en UBA y UNLP predomina la dedicación simple mientras que casi todos los docentes con dedicación exclusiva pertenecen a la Universidad Nacional de Mar del Plata. Esto se desarrolla en la misma línea que el aspecto anterior, en UBA es mucho más difícil acceder a dedicaciones más allá de la simple. Por su parte, en UNLP se ha manifestado la inadecuación de tipos de cargo y dedicaciones, es decir, que en la realidad las tareas que se realizan corresponden a otros tipos de cargos y dedicaciones.

En base a lo detallado más arriba, se puede sacar como conclusión que existen factores que han sido analizados donde no se encontraron similitudes debido a que los mismos están condicionados por las características de la universidad en la que se brindan clases. En otras palabras, existen factores que serán eminentemente diferentes debido a que están supeditados a las características (estructurales, legales, burocráticas, entre otras) de las respectivas casas de estudio.

En conclusión, se puede decir que los docentes en Bibliotecología y Ciencia de la Información que brindan clases en las universidades que poseen profesorados en la disciplina en la provincia de Buenos Aires y Ciudad Autónoma de Buenos Aires comparten un perfil común. Conociendo ese perfil es que se pueden hacer cambios para 
perfeccionarlo y fortalecerlo. Dichos cambios pueden surgir de la comunidad misma de profesionales como así también de los distintos departamentos y universidades. A lo largo del presente trabajo se ha evidenciado como estas últimas afectan la realidad de los docentes por lo que su papel (en la mejora de las formas de contratación y opciones de formación, entre otros aspectos) es crucial.

\section{Agradecimientos}

Deseo expresar mi agradecimiento a la profesora Violeta Gibaja quien me ha guiado por el proceso de investigación y a los pares evaluadores cuyas observaciones ayudaron a mejorar el presente artículo. 


\section{Q Referencias bibliográficas}

" Barber, Elsa. 2005. La enseñanza de la Bibliotecología y Ciencia de la Información: situación en las universidades argentinas. En Seminario INFOBILA como apoyo a la investigación y educación bibliotecológica en América Latina y el Caribe (2005: México). Trabajos presentados. México: Universidad Autónoma de México. Centro Universitario de Investigaciones Bibliotecológicas. p. 3-16. $<$ http://ru.iibi.unam.mx/jspui/bitstream/IIBI_UNAM/L126/1/seminario_infobila.pdf> [Consulta: 28 agosto 2016 ].

»Cabral Vargas, Brenda. 2018. El perfil docente en bibliotecología en la Escuela Nacional de Biblioteconomía y Archivonomía (México). En Bibliotecas. Vol. 36, no. 1, 1-14. <http://doi.org/10.15359/rb.36-1.2> [Consulta: 28 julio 2018 ].

» Jaramillo, Orlanda; Marisol Salazar Álvarez y María José Mercado C. 2017. Perfil del profesor de Bibliotecología-Archivística: Una mirada desde el contexto colombiano. En Información, cultura y sociedad. No. 37, 27-40. <http://revistascientificas.filo.uba.ar/index.php/ICS/article/view/3594> [Consulta: 28 julio 2018].

" Licea, Judith; Miguel Arenas; Eric González y Silvia Velázquez. 2005. La mujer docente del área de Bibliotecología en México. Presencia y Desarrollo Profesional. En Anales de Documentación. Vol. 8, 117-124. <https://revistas.um.es/ analesdoc/article/view/1501> [Consulta: 28 agosto 2016 ].

»Pirela Morillo, Johann. 2010. Los perfiles profesionales por competencias en Bibliotecología, Archivología y Ciencia de la Información. En Escalona Ríos, Lina coord. Las competencias en el perfil bibliotecológico en América Latina. Ciudad Autónoma de México: Universidad Nacional Autónoma de México. Centro Universitario de Investigaciones Bibliotecológicas. p. 1-25.

»Real Academia Española. 2016. Perfil. En Diccionario de la lengua española (23rd ed.). <http://dle.rae.es/?id=SagtYdL> [Consulta: 10 septiembre 2016].

"Silva, María Ludmila; Jonathas Luiz Silva y Maria Cleide Bernardino. 2015. Trajetória docente na Biblioteconomia da UFC-Cariri/UFCA: de 2007 a 2015. En Folha de rostro. Vol. 1, 5-19. <https://periodicos.ufca.edu.br/ojs/index.php/ folhaderosto/article/view/32> [Consulta: 28 julio 2018]. 
